\title{
Controle do Triatoma sordida no peridomicílio rural do município de Porteirinha, Minas Gerais, Brasil
}

\author{
Liléia Diotaiuti, ${ }^{1}$ Bernardino Vaz de Melo Azeredo, ${ }^{2}$ \\ Solange Cristina Uber Busek ${ }^{1}$ e Alexandre José Fernandes ${ }^{1}$
}

RESUMO O Triatoma sordida é uma espécie predominantemente peridomiciliar e, atualmente, a mais capturada no Brasil. Para melhor compreender a resposta deste triatomíneo às atividades de controle, uma pesquisa de infestação triatomínica foi realizada em outubro de 1993 em 12 localidades rurais do município de Porteirinha, MG, Brasil. Foram capturados 772 exemplares de T. sordida, dos quais 3,6\% estavam infectados pelo Trypanosoma cruzi. Dentre as 406 unidades domiciliares pesquisadas, 34,9\% estavam infestadas. Dos 695 ecótopos peridomiciliares estudados, 27,6\% foram positivos para a presença de T. sordida. O estudo revelou um peridomicílio simples, com poucos ecótopos positivos por unidade domiciliar e baixa densidade triatomínica (colônias de no máximo cinco insetos). $O$ estudo constatou uma associação entre presença de triatomíneos e ecótopos de madeira: $72,8 \%$ dos triatomíneos foram capturados neste tipo de ecótopo. Entre as unidades domiciliares positivas, 62,9\% ficavam próximas do ambiente silvestre (12 a $299 \mathrm{~m}$ ); 92,3\% dos ecótopos infestados ficavam a menos de $20 \mathrm{~m}$ das casas. O censo de animais peridomiciliares revelou que as galinhas correspondiam a 82,7\% dos animais. As unidades domiciliares infestadas foram imediatamente borrifadas com deltametrina (25 $\mathrm{mg}$ i.a. $\left./ \mathrm{m}^{2}\right)$.

Novas pesquisas de infestação foram feitas 7 meses (pesquisa 2) e 1 ano (pesquisa 3) após a borrifação. Na pesquisa 2 o número de triatomíneos correspondeu a 52,5\% da população original e, na pesquisa 3, a 79,1\% da mesma. A redução do número de triatomíneos capturados na pesquisa 2 incidiu principalmente sobre as ninfas. A grande proporção de adultos capturados nessa ocasião confirma a existência de apenas um ciclo anual do T. sordida na região. Na pesquisa 3 o aumento do número de ninfas demonstrou crescimento desta população, utilizando-se da grande disponibilidade de galinhas para alimentação. Apesar das dificuldades de borrifação e da baixa permanência do inseticida no peridomicílio, a existência de apenas um ciclo anual do triatomíneo e a lentidão na reconstituição da população original sugerem que uma borrifação anual é suficiente para controle do T. sordida. Maior eficiência poderá ser obtida com a eliminação dos esconderijos, através da substituição do material usado na construção dos anexos, principalmente a madeira.

1 Fundação Oswaldo Cruz (FIOCRUZ), Centro de Pesquisas René Rachou, CP 1743, CEP 30190-002, Belo Horizonte, Minas Gerais, Brasil. Correspondência e pedidos de separatas devem ser enviados a Liléia Diotaiuti neste endereço.

2 Fundação Nacional de Saúde, Coordenação de Minas Gerais, Belo Horizonte, Minas Gerais, Brasil.
No Brasil, a borrifação de domicílios infestados por triatomíneos pelo Programa de Controle da Doença de Chagas/Fundação Nacional de Saúde (PCDCH/FNS) reduziu a presença de triatomíneos no interior das casas. Restaram apenas pequenos focos residuais, em densidades extremamente baixas, cujo controle foi garantido pela metodologia de aplicação de insetici- 
das (com cobertura de toda a superfície das paredes internas e esconderijos debaixo ou atrás dos móveis), regularidade das borrifações e ação letal e residual dos produtos. Como resultado, a transmissão vetorial do Trypanosoma cruzi para o homem foi interrompida $(1,2)$.

Paralelamente à eliminação dos focos intradomiciliares, a infestação peridomiciliar assumiu maior importância e se tornou o maior desafio a ser enfrentado no controle de triatomíneos. Em São Paulo, dados da década de 60 já preconizavam a necessidade de repensar as estratégias de combate, uma vez que o Triatoma sordida e o Panstrongylus megistus não respondiam satisfatoriamente aos métodos de controle utilizados (3).

É importante ressaltar que a infestação peridomiciliar representa risco para a população apenas pela possibilidade de intradomiciliação dos triatomíneos. Com a crescente desmobilização do PCDCH, a partir de 1989, a intradomiciliação dos triatomíneos se tornou uma possibilidade concreta, tanto pelo aumento na densidade de triatomíneos no ambiente peridomiciliar quanto pela ocorrência de outras espécies até então tipicamente silvestres (4). A suspensão experimental da borrifação do peridomicílio (5) resultou no aumento do número de unidades domiciliares (UD) positivas e de triatomíneos capturados, o que permite concluir que a borrifação peridomiciliar deve ser mantida, principalmente para controle do tamanho das colônias.

Para isso, no entanto, muitos recursos e esforços são exigidos, já que a redução da infestação peridomiciliar chegou ao limite da técnica utilizada (borrifação). Neste contexto há que se considerar três importantes fatos: 1) as profundas modificações processadas no ambiente promoveram o deslocamento ou o desaparecimento dos refúgios e fontes de alimentação naturais dos triatomíneos, que passaram a buscar no ambiente artificial alternativa para sua sobrevivência (6). A estabilidade do ambiente artificial (muitos esconderijos e fartura alimentar) permite a explosão da população triato- mínica, que alcança densidades muito altas quando comparadas com as populações presentes no ambiente silvestre; 2) os inseticidas se degradam com mais rapidez no peridomicílio por ação da luz, vento, umidade e calor, com conseqüente redução da proteção contra reinfestações por insetos vindos do ambiente silvestre ou sobreviventes das borrifações anteriores (7); 3) a complexidade do próprio peridomicílio pode dificultar a deteç̧ão da infestação e seu controle químico.

O estudo descrito no presente artigo teve como objetivo conhecer melhor o peridomicílio e identificar os fatores que favorecem sua colonização no norte do Estado de Minas Gerais, área predominantemente infestada pelo $T$. sordida. Além disso, o estudo buscou avaliar o impacto das ações de controle (borrifação) sobre a população de triatomíneos peridomiciliares. Por peridomicílio entende-se a área em torno da habitação afetada pela atividade humana, conforme a definição ecológica de Bos (8).

\section{MATERIAIS E MÉTODOS}

O município de Porteirinha, norte de Minas Gerais, é constituído por 251 localidades e tem como principais atividades econômicas a plantação de algodão e a pecuária. Doze localidades, caracteristicamente rurais (com até $50 \mathrm{UD}$ ), foram submetidas, em outubro de 1993, à uma pesquisa de infestação triatomínica (pesquisa 1). A pesquisa foi realizada por uma equipe da FNS (9). O intradomicílio, todos os ecótopos e construções anexas do peridomicílio foram investigados quanto à presença de triatomíneos. Os formulários utilizados identificavam a unidade domiciliar (número da casa) e o local onde os triatomíneos foram capturados (intra ou peridomicílio), a medida em $\mathrm{m}^{2}$ onde se incluía o peridomicílio, a distância do peridomicílio ao ambiente silvestre e entre as habitações mais próximas, tipos e características dos ecótopos peridomiciliares e animais presentes no peridomicílio no momento da pesquisa. Foram estuda- das todas as 406 UD existentes nas localidades. Todo o município vem sendo regularmente incluído nas atividades da FNS, ou seja, borrifação das UD positivas.

Todos os triatomíneos encontrados foram capturados e enviados ao Laboratório de Biologia de Triatomíneos e Epidemiologia da Doença de Chagas/Centro de Pesquisas René Rachou para identificação quanto à espécie e estádio evolutivo e para pesquisa de infecção pelo T. cruzi. O conteúdo estomacal de 612 insetos foi submetido à reação de precipitina para identificação da fonte alimentar, utilizando-se soros anti-proteínas séricas de homem, gato, cão, gambá, ave, roedor, cabra e porco. Imediatamente após a captura procedeu-se à borrifação das UD infestadas com deltametrina (25 mg i.a. $\left./ \mathrm{m}^{2}\right)$.

Em maio de 1994, 7 meses depois da pesquisa 1 , nova pesquisa de infestação foi realizada (pesquisa 2); finalmente, depois de 1 ano, em outubro de 1994, repetiu-se uma terceira pesquisa (pesquisa 3). Para avaliação da densidade triatomínica, a mesma técnica de captura foi utilizada para as três pesquisas triatomínicas.

A caracterização da infestação peridomiciliar foi realizada a partir dos dados obtidos na pesquisa 1. Para avaliação do impacto da borrifação sobre a população de triatomíneos peridomiciliares utilizaram-se os dados das pesquisas 1, 2 e 3 . A comparação dos números de triatomíneos capturados foi feita através do teste do qui-quadrado $\left(\chi^{2}\right)$.

\section{RESULTADOS}

\section{Caracterização da infestação peridomiciliar}

Os resultados da pesquisa 1 , de outubro de 1993, revelaram que dentre as $406 \mathrm{UD}$ pesquisadas $142(34,9 \%)$ estavam infestadas por triatomíneos. Um total de $772 \mathrm{~T}$. sordida foram capturados (173 adultos e 599 ninfas).

Foram observadas pequenas e raras colônias intradomiciliares, representa- 
das por apenas 12 triatomíneos capturados neste ambiente. A maioria dos triatomíneos foi capturada no peridomicílio $(97,9 \%)$, com taxa de infecção pelo $T$. cruzi de $3,6 \%$.

Foram examinados 695 ecótopos peridomiciliares (média de ecótopos por UD igual a $4,5 \pm 3,2$; mediana e moda iguais a 4). Destes, $192(27,6 \%)$ foram positivos para a presença de triatomíneos. Dentre as colônias, $89,4 \%$ eram constituídas por um ou dois triatomíneos e 10,6\% eram constituídas por três a cinco triatomíneos. Não foram detectadas colônias constituídas por mais de cinco insetos. Os ecótopos mais freqüentemente infestados foram montes de madeira $(100 \%)$, cercas $(100 \%)$, árvores $(85,7 \%)$, montes de telhas $(57,5 \%)$, galinheiros $(53,2 \%)$ e chiqueiros $(28,1 \%)$. Dos triatomíneos capturados, $72,8 \%$ encontravam-se em ecótopos constituídos por madeira; 10,2\% em ecótopos constituídos por barro; os demais triatomíneos foram capturados em montes de telhas ou tijolos $(10,4 \%)$ ou construções de adobe $(1,5 \%)$ (não obtivemos informações sobre infestação em 5,1\% dos ecótopos estudados).

O peridomicílio está a uma distância média do ambiente silvestre de $279 \pm$ $332 \mathrm{~m}$. A distância varia de 12 a $299 \mathrm{~m}$ $(63,2 \%) ; 300$ a $599 \mathrm{~m} \mathrm{(26,5 \% );} \mathrm{e} 600$ a $2000 \mathrm{~m}(10,3 \%)$, sendo as unidades domiciliares mais próximas as mais freqüentemente infestadas $(62,9 \% ; 22,7 \%$; e $14,4 \%$, respectivamente). Também é variável a área média que compreende o peridomicílio $\left(1826 \pm 2996 \mathrm{~m}^{2}\right)$, correspondendo $73,2 \%$ a medidas que variaram de 400 a $2999 \mathrm{~m}^{2}$. A avaliação da distância entre os anexos ou ecótopos positivos e a casa demonstra que $92,3 \%$ deles estão a menos de $20 \mathrm{~m}$ (média de 12,2 $\pm 7,6 \mathrm{~m}$ ).

A área média compreendida pelo peridomicílio também era variável $\left(1826 \pm 2996 \mathrm{~m}^{2}\right)$. Dentre os peridomicílios analisados, 73,2\% tinham área entre 400 e $2999 \mathrm{~m}^{2}$. O estudo revelou que $92,3 \%$ dos anexos ou ecótopos positivos estavam a menos de $20 \mathrm{~m}$ de distância das casas (média de 12,2 \pm 7,6 m).

$\mathrm{O}$ censo de animais presentes no peridomicílio no momento da captura revelou uma população de 4352 indivíduos. As galinhas correspondiam a $82,7 \%$, porcos a $10,1 \%$, cães a $3,1 \%$ e gatos a $2 \%$. Estes mesmos animais estavam presentes em 89,4\%; 76,1\%; $59,9 \%$ e $43,7 \%$, respectivamente, das UD estudadas. A média de animais por UD foi de 25,3 galinhas; 3,1 porcos; 1 cão; e 0,6 gatos. Estes animais perfaziam 97,9\% do total observado. Uma pequena e variada população de outros animais também foi observada, constituída por cabras, pombos, pato, peru, pássaros ornamentais, coelhos, carneiro, veado, preá e cavalo.

Dos 772 triatomíneos capturados, $160(21 \%)$ não continham no tubo digestivo material suficiente para processamento da reação de precipitina. Para os 612 triatomíneos analisados, $20,4 \%$ não reagiram com os anti-soros testados. Dentre os que reagiram, 74,5\% apresentavam conteúdo alimentar procedente de um único hospedeiro. A ocorrência de fonte dupla foi de $4,9 \%$; e de fonte tripla, 0,2\%. Em 422 $(69 \%)$ amostras as reações foram positivas com soro anti-ave; em $22(3,6 \%)$, com soro anti-cão; em 19 (3,1\%), com anti-roedor; em 18 (2,9\%), com antiporco; em $3(0,5 \%)$, com anti-gambá; em $2(0,3 \%)$, com anti-homem; e em 1 caso $(0,2 \%)$ com soro anti-gato. $\mathrm{Ne}-$ nhuma amostra reagiu com o anti-soro de cabra.

\section{Estrutura da população triatomínica peridomiciliar antes e depois da borrifação}

A distribuição dos exemplares de T. sordida capturados, conforme estádio evolutivo, está apresentada na tabela 1. Sete meses após a borrifação observou-se que a população de triatomíneos encontrada representava $52,5 \%$ da população original. Um ano depois, a população original ainda não havia sido reconstituída, representando os insetos capturados 79,1\% da mesma. A soma dos triatomíneos capturados nas pesquisas 2 e 3 correspondeu a 1016 insetos, número estatisticamente superior ao encontrado na pesquisa 1. A análise da estrutura etária revela que a redução no número de triatomíneos capturados na pesquisa 2 incidiu principalmente sobre os estádios ninfais. O número de adultos permaneceu praticamente inalterado nas três pesquisas (teste do $\chi^{2}$ quadrado).

\section{DISCUSSÃO E CONCLUSÕES}

A maioria dos peridomicílios estudados $(73,2 \%)$ correspondiam a áreas com medidas entre 400 e $2999 \mathrm{~m}^{2}$, onde se concentravam criações de animais, pomar, horta, áreas de despejo e armazenamento, cuja proximidade da casa facilitava o trabalho doméstico (principalmente da mulher). Não é surpreendente, portanto, que 92,3\% dos ecótopos infestados estejam a menos de $20 \mathrm{~m}$ de distância da casa.

A borrifação anual das UD positivas tem mantido a infestação peridomiciliar baixa, o que pode ser confirmado pelos dados de densidade triatomínica aqui apresentados, pois, de modo geral, as colônias eram pequenas, com densidade em torno de um ou dois insetos. No entanto, a maior infestação das casas mais próximas do ambiente silvestre sugere um fluxo de recolonização do ambiente artificial por triatomíneos procedentes de ecótopos naturais.

Os resultados demonstram forte associação entre $T$. sordida, madeira e galinhas. O T. sordida é encontrado na natureza sob cascas secas de árvores (5, 6); as galinhas, por sua vez, estão em toda a parte no quintal, em árvores, galinheiros, paióis, chiqueiros, currais e cercas, feitos de madeira. É de se esperar, portanto, que a maioria dos insetos sugue sangue de ave, conforme verificado anteriormente (10) e confirmado no presente estudo através da reação da precipitina (69\% das amostras foram positivas para soro antiave). $\mathrm{O}$ pequeno número de insetos que se alimentam de outras fontes que não sejam as aves reforça a preferência ornitofílica do T. sordida. Este fato pode estar relacionado à dificuldade de adaptação do T. sordida a mamíferos e, conseqüentemente, ao intradomicílio. 
TABELA 1. Número de exemplares de Triatoma sordida, conforme o estádio evolutivo, capturados nas três pesquisas realizadas no município de Porteirinha, Minas Gerais, Brasil, 1993-1994

\begin{tabular}{cccc}
\hline Estádio & $\begin{array}{c}\text { Pesquisa 1 } \\
\text { (outubro/1993) }\end{array}$ & $\begin{array}{c}\text { Pesquisa 2 } \\
\text { (maio/1994) }\end{array}$ & $\begin{array}{c}\text { Pesquisa 3 } \\
\text { (outubro/1994) }\end{array}$ \\
\hline I & 14 & 8 & 87 \\
II & 51 & 17 & 70 \\
III & 111 & 37 & 114 \\
IV & 164 & 39 & 93 \\
V & 259 & 104 & 75 \\
Adultos & 173 & 200 & 172 \\
Total & 772 & 405 & 611 \\
\hline
\end{tabular}

Gambás e roedores, hospedeiros de reconhecida importância como elo de ligação dos ciclos silvestre e peridomiciliar do T. cruzi (11), podem ter também em Porteirinha um papel importante na infecção dos triatomíneos peridomiciliares. Por outro lado, a associação observada pelo presente estudo com outros animais, como cães, gatos e porcos, deve ser melhor estudada, já que a taxa de infecção encontrada $(3,6 \%)$ foi bem mais alta que a taxa que há muitos anos vem sendo observada no estado de Minas Gerais $(0,5 \%)(12)$.

Os resultados do estudo evidenciam a extrema simplicidade dos peridomicílios analisados, especialmente quando comparados a outras áreas, o que retrata a pobreza da região. Estudos preliminares na região de Minas Gerais conhecida como Triângulo Mineiro demonstram um peridomicílio muito mais complexo, com densidades triatomínicas (cerca de 13 triatomíneos por peridomicílio) mais altas, podendo atuar como barreira contra a infestação intradomiciliar (4). Da mesma forma, o manejo do peridomicílio de Porteirinha, com melhoria do padrão dos anexos, retirada de entulhos e afastamento dos triatomíneos das fontes de alimentação não seria operacionalmente difícil se não fosse o contexto social tão adverso ao aporte educativo e de participação popular.

A borrifação realizada ao final da pesquisa 1 serviu para diminuir o número de exemplares de $T$. sordida nos peridomicílios estudados. $\mathrm{O}$ im- pacto da borrifação foi maior sobre os estádios ninfais dos triatomíneos. O número de adultos capturados nas três pesquisas praticamente não foi alterado. Como não há evidências de menor suscetibilidade dos triatomíneos adultos à deltametrina, supomos que parte deles morreu e que a população foi reconstituída a partir das ninfas que sobreviveram e de exemplares provenientes do ambiente silvestre. Paralelamente, a migração de exemplares adultos peridomiciliares para o ambiente silvestre também deverá colaborar para a estabilidade no vado em área próxima (11). Este fluxo de insetos parece ocorrer em ambas as direções, tanto do peridomicílio para o ambiente silvestre, como do ambiente silvestre para o peridomicílio. A grande proporção de adultos capturados em maio de 1994 (49,4\% do total de insetos capturados) confirma na região a existência de um pico imaginal no final do primeiro semestre do ano, conforme verificado em outras áreas $(13,14)$, e a existência de apenas um ciclo anual do T. sordida.

O grande número de ninfas $\mathrm{V}$ capturadas na pesquisa 2 corresponde, em parte, às ninfas I a IV que sobreviveram à borrifação. Na avaliação realizada em outubro de 1994 (pesquisa 3), o aumento no número dos diferentes estádios ninfais demonstra claramente o crescimento da população de $T$. sordida, que utiliza a grande disponibilidade de galinhas como principal fonte de alimentação. $\mathrm{O}$ número de ninfas I número de adultos, conforme obser- capturadas em outubro de 1994 pode indicar aumento do potencial de recuperação da população após a redução da mesma através da borrifação - a diminuição da população altera o número de triatomíneos por fonte alimentar, o que diminui a competição para obtenção de repasto, conforme observado com o T. infestans (15). Provavelmente, caso os insetos não tivessem sido retirados dos seus ecótopos na pesquisa 2, a população triatomínica teria se reconstituído em termos numéricos (sem levar em conta a estrutura populacional) ao longo do ano estudado. As observações anteriores são compatíveis com os resultados obtidos no estado de São Paulo sobre a ocorrência de um longo ciclo evolutivo do T. sordida em condições de campo (468 dias) (14).

A sobrevivência de triatomíneos à borrifação confirma a limitação desta técnica na eliminação completa dos insetos peridomiciliares. Tal limitação é justificada pela dificuldade que representam para a borrifação o número e variedade de ecótopos onde se abrigam os triatomíneos, além da transitoriedade da ação residual de inseticidas (em especial os biodegradáveis) neste ambiente. Daí a possibilidade de sobrevivência de uma parte da população de triatomíneos, que permanece protegida contra a ação do inseticida, principalmente em cercas, galinheiros, chiqueiros, etc., feitos de madeira. Mesmo assim, o fato de o ciclo do triatomíneo ser anual, além da lentidão na reconstituição da população em geral, indicam que uma borrifação em intervalos próximos a 1 ano será suficiente para controlar as populações peridomiciliares do $T$. sordida. Somado a isso, maior eficiência poderá ser obtida com a eliminação dos esconderijos, através da substituição do material usado na construção dos anexos, principalmente a madeira.

Agradecimentos. Os autores agradecem à equipe de campo da Fundação Nacional de Saúde, em especial a João Geraldo Resende, chefe do setor técnico do distrito de Montes Claros. 


\section{REFERÊNCIAS}

1. Dias JCP. Control of Chagas disease in Brazil. Parasitol Today 1987;3(11):336-341.

2. Silveira AC, Rezende DF. Epidemiologia e controle da transmissão vetorial da doença de Chagas no Brasil. Rev Soc Bras Med Trop 1994;27(supl III):11-22.

3. Silva EOR, Dias Jr J, Guarita OF. Suspensão do rociado no combate ao Triatoma infestans em áreas do estado de São Paulo, Brasil. Rev Saude Publica 1969;3:173-181.

4. Diotaiuti L. Importância atual e perspectivas de controle do Triatoma sordida em Minas Gerais [tese de doutorado]. Belo Horizonte: Universidade Federal de Minas Gerais; 1991.

5. Diotaiuti L, Carneiro M, Loiola CCP, Silveira Neto HV, Coutinho RM, Dias JCP. Alternativas de controle do Triatoma sordida no Triângulo Mineiro, I: Borrifação parcial (intradomicílio) no município de Douradoquara, MG, Brasil. Rev Soc Bras Med Trop 1988;21(4): 199-203.

6. Forattini OP. Biogeografia, origem e distribuição da domiciliação de triatomíneos no Brasil. Rev Saude Publica 1980;14:265-299.

7. Oliveira Filho AM. New alternatives for the control of triatomines in peridomestic build- ings. Rev Soc Bras Med Trop 1989;22(supl II): 53-57.

8. Bos R. The importance of peridomestic environmental management for the control of the vectors of Chagas Disease. Rev Argent Microbiol 1988;20(supl):58-62.

9. Brasil, Ministério da Saúde/SUCAM/ DIDOCH. Manual de normas técnicas da campanha de controle da doença de Chagas. Brasília: Centro de Documentação do Ministério da Saúde; 1980.

10. Forattini OP, Barata JMS, Santos JLF, Silveira AC. Hábitos alimentares, infecção natural e distribuição de triatomíneos domiciliares na Região Central do Brasil. Rev Saude Publica 1982;16:171-204.

11. Diotaiuti L, Pereira AS, Loiola CF, Fernandes AJ, Schofield CJ, Dujardin JP, et al. Interrelation of sylvatic and domestic transmission of Trypanosoma cruzi in areas with and without domestic vectorial transmission in Minas Gerais, Brazil. Mem Inst Oswaldo Cruz 1995;90(4):443-448.

12. Diotaiuti L, Paula OR, Falcão PL, Dias JCP. Evaluation of Chagas Disease vector control programs in Minas Gerais, Brazil, with special reference to Triatoma sordida. Bol Oficina Sanit Panam 1994;28(3):211-219.

13. Diotaiuti L, Loiola CF, Falcão PL, Dias JCP. The ecology of Triatoma sordida in natural environments in two different regions of the state of Minas Gerais, Brazil. Rev Inst Med Trop Sao Paulo 1993;35(3):237-245.

14. Forattini OP, Ferreira OA, Rabello EX, Barata JMS, Santos JLF. Aspectos ecológicos da tripanosomíase americana, XVIII: Desenvolvimento e ciclos anuais de colônias de Triatoma infestans, T. sordida e Rhodnius neglectus em ecótopos artificiais, no ambiente peri e extradomiciliar. Rev Saude Publica 1983;17:243-262.

15. Schofield CJ. The biosystematics of Triatominae. Em: Service MW, ed. Biosystematics of haematophagous insects. Oxford: Systematics Association, Clarendon Press; 1988:248-312.

Manuscrito recebido em 30 de julho de 1996. Aceito em versão revisada em 22 de abril de 1997.
ABSTRACT

\section{Control of Triatoma sordida in the rural peridomestic environment of Porteirinha municipality, Minas Gerais, Brazil}

Triatoma sordida, a predominantly peridomestic species, is currently the triatomine species most frequently collected in Brazil. To evaluate the effectiveness of control activities against this species, a survey to determine infestation rates was carried out in October 1993 in 12 rural localities in the municipality of Porteirinha, State of Minas Gerais, Brazil. In the survey, 772 T. sordida were captured, of which 3.6\% were infected with Trypanosoma cruzi. Of the 406 dwellings investigated, 34.9\% were infested, and $27.6 \%$ of 695 peridomiciliar ecotopes contained $T$. sordida. The area around the dwellings was relatively uniform, with few positive ecotopes per household and low triatomine densities (a maximum of five insects per colony). The study found an association between the presence of insects and wood: $72.8 \%$ of the insects were captured in this type of ecotope. The majority of the positive households $(62.9 \%)$ were close to forests (12 to $299 \mathrm{~m}$ ); $92.3 \%$ of the infested ecotopes were less than $20 \mathrm{~m}$ from the house. A census of peridomestic animals revealed that chickens were the most abundant $(82.7 \%)$. All positive households found in the survey were sprayed immediately with deltamethrin $\left(25 \mathrm{mg}\right.$ a.i. $\left./ \mathrm{m}^{2}\right)$.

New surveys were carried out 7 months (survey 2) and 12 months (survey 3) after spraying. In survey 2 , the number of insects collected corresponded to $52.5 \%$ of the original total, and in survey $3,79.1 \%$. The decrease in population observed in survey 2 was mainly due to a reduction in the population of nymphs. The large proportion of adults captured in that survey supports the idea that $T$. sordida produces only a single generation per year in this region. In survey 3 the increase in the number of nymphs, attributable to the great availability of chickens as a source of food, resulted in population growth. Despite the difficulties of spraying and the short persistence of the insecticide, the slow population response of $T$. sordida suggests that one annual spraying is sufficient for effective control of this species. Higher effectiveness could be obtained by eliminating peridomestic hiding places, for example, by replacing wooden annexes with structures made from other materials. 